\title{
A Study on Computational Process in Gene Expression Data
}

\author{
E.Monica Sushil Cynthia ${ }^{* 1}$ VairaprakashGurusamy ${ }^{\# 2}$ S.kannan $^{\# 3}$ \\ ${ }^{1}$ Department of MCA, American College, Madurai, Tamilnadu, India. \\ ${ }^{1}$ Monicasushil777@yahoo.com \\ ${ }^{2,3}$ Department of Computer Applications, Madurai Kamaraj University, Madurai, Tamilnadu, India. \\ ${ }^{2}$ vairaprakashmca@gmail.com \\ ${ }^{3}$ skannanmku@gmail.com
}

\begin{abstract}
This context is commenced to examine the various methods and its challenges in Disease Identification of Gene Expression Data.The elementalresponsibility of these techniques is classification and categorization of gene expression, analysis of the expression, Pattern Recognition, and Identification. This provides an inclusive survey of Micro Array Data analysis techniques and intends a processing component for disease identification. For thehealthcare provider, it is essential to maintain the quality of data because this data is useful to provide cost effective healthcare treatments to the patients. Health Care Administration retains the Microarray data which is refined by expertise and is analyzed by the expertise to identify the disease. This process of analyzing this Microarray data as manual is complicated in identification and classification; due to this Microarray data some difficulties such as missing information, empty values, and incorrect entries. Exclusive of quality information there is no valuableconsequences. For successful data mining, animpediment in health data is individual the majordifficulty for examining medical data. So, it is essential to maintain the quality and accuracy data for data mining to making aneffective decision. The major goal of this survey is focused on various techniques of data mining for developing a prediction model for disease susceptibility using Gene Expression Data.The microarray data is pre-processed to analyze the gene expression to classify the overexpression and under-expression data. Then the classified gene data is then clustered and the best feature selection is applied to discover a pattern. Finally, the association mining handled under the organized set of the gene expression data to theidentification of the disease. This context provides efficient techniques to overcome the manual identification of diseases.
\end{abstract}

Keywords: Gene Expression Data, Disease Identification, Classification, Association Mining,Pattern Recognition

\section{Introduction}

DNA microarrays propose the capability to appear at the expression of thousands of genes in a particularresearch one of the significantrelevance of microarray knowledge is disease identification and classification. Throughmicroarraytechnology, researchers will be proficient in organizing special diseases according todissimilar expression intensity incommon anddevelopment cells, to determine the affiliationamong genes, to recognize the critical genes in the development of disease [1]. The main task of microarray classification is to construct a classifier from chronological microarray gene expression data, and then it utilizes the classifier to categorizeprospectapproachingdata. Appropriate to the rapid improvementofDNA microarray knowledge, gene rangetechniques andorganizationtechniques are being figured for enhanceduse of classification algorithm in microarray gene expression data.The study of outsized gene expression data sets is fetching a dispute in disease classification [2]. Thusgene selection is one of the significantcharacteristics. Proficient gene selection can considerablysimplicity computational burden of the consequent classification assignment and can yield a much smaller and more condensed gene set,not including thedefeat of classification. In classifying microarray data, the key objective of gene selection is to explore for the genes, which remain the greatest amount of information about the set and decrease the categorization error. [3] Data mining techniques classically descend into either supervised or unsupervised classes.Microarray technologies afford a dominant tool by which the expression prototypes of thousands of genes can be examinedconcurrently whose relevancecollection from disease diagnosis to treatment response. Gene expression is the renovation of the DNA progression into mRNA progression by dictation then transformed into amino acid sequences called proteins.

The key challenge in classifying gene expression data is the annoyance of dimensionality difficulty. There is ahugeamount of genes (features) evaluated to small sample sizes [3]. To conquer this, feature selection is worn to recognize differentially articulated genes and to eliminateinappropriate genes. Gene selection remains asa significant task to extend the exactness and speed ofclassification structures.In general, feature selection can be prepared into three kinds: Filter, Wrapper, and Embedded methods. They are classified based on how afeature selection methodmerges with the production of aclassification form. Anextensivequantity of literature has been available on gene selection techniques for constructionvaluable classification model. In this paper, we 
present a review of feature selection techniques for disease identification and classification.In this study, it has been focused on Microarray gene association analysis from anassociation mining approach.

\section{Challenges in Analyzing Microarray Data}

Numerous challenges in microarray require to be atreatywithpreviousto new information about gene expression can be exposed. Some of the problems are:

1. Microarray data is high dimensional data illustrated by thousands of genes in only someexample sizes, which reasonimportant problems such as inappropriate and blast genes, difficulty in creating classifiers [4], and severalmisplaced gene expression values due to indecentexamining. In addition, most of thestudies that functional microarray data are endured from information overfitting, which involvesextrasupport.

2. Mislabeled records or difficult tissues product by specialist also another form of negative aspect that could reduce the accuracy of experimental results and direct to anindefinite conclusion about gene expression patterns $[4,5]$.

3. Biological relevanceproduct is another essentialcondition that should be inuse into adescription in consideringmicroarray data rather than only focusing on theexactness of disease classification [6]. Even though there is no suspiciongaining high accuracy classification consequences are significant in microarray data study, but revealing thegenetic information through the development of categorization is also important.

4. Cross-platform evaluation of gene expression studies iscomplex to perform when microarrays were build using specialprinciples. Thus, the consequences cannot be imitated.

\section{Processing Components}

Disease Identification and classification is based on microarray data analysis classical methods like preprocessing, clustering, feature selection, pattern discovery, classification and association mining. In following sections, surveys of existing methods are analyzed. In the remaining sections, the importance of processing modules is discussed.

\section{Overview of the existing techniques in disease identification}

Feature selection methodsin excess of DNA microarray focus on filter techniques. The majority of the proposed techniques are univariate i.e. Each feature is measured individually. The featuresignificanceachieve is considered, and low attaining features aredistant [8]. The top ordered genes are used to construct the classifier. Following are the feature selection methods.

(a) Information Gain (IG)

Entropy assessment is used in Information Gain, Gain Ratio, and Symmetrical improbability aspect grading methods [8]. Entropy of Y is

$$
H(Y)=-\sum_{y \in Y} p(y) \log _{2}(p(y))
$$

$\mathrm{p}(\mathrm{y})$ is the trivial probability soliditypurpose for unsystematic variable $\mathrm{Y}$. the provisional entropy of $\mathrm{Y}$ after examining $\mathrm{X}$ is

$$
H(Y \mid X)=-\sum_{x \in X} p(x) \sum_{y \in Y} p(y \mid x) \log _{2}(p(y \mid x))
$$

$\mathrm{p}(\mathrm{y} \mid \mathrm{x})$ is the provisional probability of yspecified $\mathrm{x}$. The information gained regarding $\mathrm{Y}$ after surveying $\mathrm{X}$ is

$$
I G=H(Y)-H(Y \mid X)
$$

Information gain is symmetrical measure,

$$
I G=H(Y)-H(Y \mid X)=H(X)-H(X \mid Y)
$$

(b) Gain Ratio (GR)

In order to expect $Y$ standardize information gain (IG) byisolating it by theentropy of $X$. The Gain_ratio is

$$
\text { Gain_ratio }=\frac{I G}{H(X)}
$$


The gain ratio is an asymmetrical evaluate. Gain ratio falls in therange $[0,1]$ since of normalization. Gain_ratio $=1$ specifythat Xentirely predicts $Y$ and Gain_ratio $=0$ designate that $X$ and $Y$ are independent.

(c) Fisher's Criteria

The equation used to rank the genes in fisher criteria is

$$
\text { fisher }(g)=\frac{\left(m_{1}(g)-m_{2}(g)\right)^{2}}{s_{1}^{2}(g)+s_{2}^{2}(g)}
$$

$\mathrm{m}_{1}$ and $\mathrm{m}_{2}$ stand for the mean expression value of thej ${ }^{\text {th }}$ gene in excess ofall samples in cancer and standardcasing respectively. $\mathrm{s}_{1}$ and $\mathrm{s}_{2}$ indicate the standard deviation of thej ${ }^{\text {th }}$ gene over all samples inexaggerated and common case respectively[9].

(d) Clustering and Network analysis based Technique

Shang Gao, Omar Addam and colleagues in [10] projectedthese two feature selectionsystems. The proposedclustering based process uses Genetic Algorithm (GA) approach.

(e) Support Vector based Correlation Coefficient (SVcc)

This method chosesustain vector data spots using support vector machine (SVM). These preferred data points are extra used for ranking the genes using association coefficient. The top ordered genes are used for categorization.

Classification and clustering are in commonmeasured asalike; the only variation is classification is asupervised learning techniquewhereas clustering is an unsupervised learningprogression. In classification, the category label of each training tuple is recognized in progress hence called as supervised learning. The classifier constructsusing the known instances (training set) to effectivelyforecast the group of thenewinstance (test set). The exactness of the classifier is resolute as the proportion of test tuples that are properly classified by the classifier from the test set. In other words, the classification is all aboutforecasting the class of the new instance by learning fromidentified instances and their class labels. KNN, DT, SVM, NN, NB are classification technique [4], [6], [8].

\section{Microarray Data Analysis}

Microarray datais the center of arevolution in biotechnology. It is used to monitor the expression of tens ofthousands of genes at the same time. Hence it isused toaccomplish manygenetic tests in parallel [10]. The result of amicroarray experiment is alist of genes that are found to bedifferentially expressed in specialkinds of tissues. The microarray dataset can be variety of an $\mathrm{M} \mathrm{x} \mathrm{N}$ matrix $\mathrm{D}$ of expression values, where the row stand for genes g1, g2, g3 ..., in and the column correspond to different experimental conditions s1, s2, s3 ... sn. Each aspect $\mathrm{D}[\mathrm{i}, \mathrm{j}]$ represents the expression stage of the gene gi in the sample so.The matrix typicallyholdsa large amount of data, so data mining techniques are used to extort usefulinformation.

\section{Pre-processing}

Data pre-processing in microarray expertise is anessentialpreliminarystepprevious to data investigation is achieved. Various pre-processing techniques have been projected but nothing has confirmedperfect to date. Regularly, datasets are inadequate by laboratory restraints so that therequire is for strategy on feature and toughness, to notifyadvancetestingalthough data are yet classified.Another goal of preprocessing is to "clean" the raw data. The measured intensities are not only influenced by the actual RNA abundance but also by other sources of variation. Commonly $[10,11]$, preprocessing microarray data is a three-step procedure: background correction, normalization, and summarization. Therefore, many researchers developed alternative algorithms for each of the three preprocessing steps. 


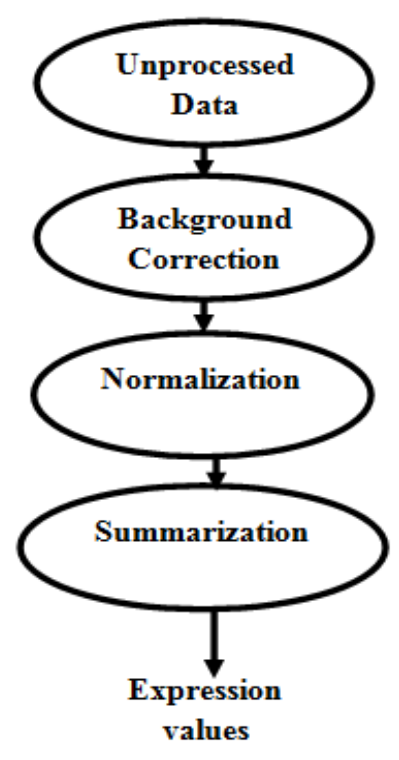

Figure 2: Microdata Pre-processing

The algorithms are implemented such that every step is self-contained, i. e. every method for one step can combine with every method for another step opening a large playground for all the combinatory. The number of possible combinations is even higher since the first three steps are optional; only summarization is mandatory to complete the preprocessing.

\section{a) Background Correction}

Background correction methods estimate the background portion of the probe signals and subtract it accordingly. In the case of DNA arrays, thebackground can be estimated from the area surrounding and separating the spots.

\section{b) Normalization}

In general, the initial transformation useful to expression data, referred to as normalization, regulates the entity hybridization intensities to stabilitythem suitablyso that significantgeneticassessment can be made [11]. There is a quantity of basis why information must be standardized, including irregularmeasured of preliminary RNA, distinctions in classification or recognitionefficiencies involving theluminouscolorantused, and methodical biases in the considered expression stages. Abstractly, normalization is related to regulating expression levelsconsidered by northern investigation or quantitative repealtranscript relative to the expression of one or more reference genes whose levels are assumed to be constant between samples.

\section{c) Summarization}

The process of reducing multiple measurements on the same gene down to a single measurement by combining some manner.

\section{Expression Analysis}

The standard genetic approach for investigating biological typically begins by recognizingtransformations that source a phenotype of importance [14]. Over-expression or Under-expression of a wild-type gene product, though, can also basisdistorted phenotypes, afforded that geneticists with another yet influentialdevice to categorize components that mayresideafterdisregarded using established loss-of-function study. The most admired two types of Differential gene expression conferred as follows

\section{Statistical tests}

a) Statistical t-test: a two illustrationposition test of the null suggestion that the $\theta$ means of two usuallycirculated populations are equivalent.

b) Welch's t-test: asymmetricalvariation

c) Mann-Whitney U test (also called Wilcoxon rank-sum test): $\theta$ nonparametric

\section{U-testv}

a) Robustness: U-test is furthertough to outliers

b) Effectiveness: When familiarity holds, the effectiveness of the U-test is as regards 0.95 when evaluated to the t-test. For distributions adequatelydistant from standard and for suitablyhuge sample sizes, the U-test can be greatly more proficient than the t-test. 


\section{Clustering}

However, the huge number of genes and the difficulty of genetic seriouslyenhance the challenges of understanding and inferring the resultantgroup of data, which frequently consists of millions of dimensions [14, 15]. Aninitial step to addressing this disputing is the use of clustering methods, which is important in the information mining procedure to exposeordinary structures and recognize amotivatingpattern in the essential data. Cluster analysis inquires about to separation a given data set into collections based on particular features so that the data endsin a group are more related to every other than the ends in dissimilar groups [15]. Anextremely rich literature on cluster analysis has developed more than the past three decades.

Clustering techniques have established to be supportive to appreciate gene function, gene regulation, cellular processes, and subtypes of cells. Genes with related expression prototypes (co-expressed genes) can be clustered collectively with related cellular functions. Moreover, co-expressed genes in the equivalent cluster are probably to be concerned in the same cellular progressions and a strong association of expression patterns among those genes specifies co-regulation. Penetrating for general DNA sequences at the supporterexpanses of genes in the same cluster permits regulatory motifs detailed to every gene cluster to be recognized and regulatory basics to be proposed.

Table 2: Clustering Techniques Comparison Table

\begin{tabular}{|c|c|c|}
\hline Techniques & Advantages & Disadvantages \\
\hline K-Means Clustering & $\begin{array}{l}\text {-Less Time Complexity } \\
\text {-Less Space Complexity } \\
\text {-Order-independent }\end{array}$ & $\begin{array}{l}\text {-More iteration for optimal no. } \\
\text { of clusters } \\
\text {-Sensitive to noise when huge } \\
\text { noise occurs }\end{array}$ \\
\hline Hierarchical Clustering & $\begin{array}{l}\text {-Embedded Flexibility } \\
\text {-Ease of handling of any forms } \\
\text {-More versatile }\end{array}$ & $\begin{array}{l}\text {-Difficult to modify } \\
\text {-Changes may cause huge } \\
\text { complexity } \\
\text {-High Computational } \\
\text { Complexity }\end{array}$ \\
\hline $\begin{array}{l}\text { Self-organizing } \\
\operatorname{map}(\mathrm{SOM})\end{array}$ & $\begin{array}{l}\text {-Combination of several map units allows the } \\
\text { construction of non-convex clusters } \\
\text {-Measuring and joining criteria I useful for big } \\
\text { clusters } \\
\text {-Sub-optimal partition provided if the initial } \\
\text { weights are not properly chosen }\end{array}$ & $\begin{array}{l}\text {-Not much effective if the } \\
\text { pattems are merged } \\
\text {-Not effective in highly noisy } \\
\text { data }\end{array}$ \\
\hline $\begin{array}{l}\text { Expectation- } \\
\text { maximization Clustering }\end{array}$ & $\begin{array}{l}\text {-Robust to noisy data } \\
\text {-Handle high dimensional data } \\
\text {-Converges fast } \\
\text {-Linear in data size } \\
\text {-Accept desired no. of clusters }\end{array}$ & $\begin{array}{l}\text {-Provide less accuracy in some } \\
\text { noisy data } \\
\text {-Very sensitive for noise } \\
\text { - Low quality results when } \\
\text { using random dataset }\end{array}$ \\
\hline
\end{tabular}

(a) K-Means Clustering

The k-means algorithm [15] is one of the most extensively used methods for clustering. It begins by initializing the $\mathrm{k}$ cluster midpoint, where $\mathrm{k}$ is resolute prior to clustering. Then, each object (input vector) of the data set is assigned to the cluster whose center is the nearest.

\section{(b) Hierarchical Clustering}

Partitioning algorithms are based on identifying an original number of collections, and iteratively reallocating objects betweensets to convergence. In distinguishing, hierarchical algorithms merge or split existing sets, generating a hierarchical construction that reproduces the classification in which groups are combined or divided. This method iterates in anticipation of all objects are in a particular group. The differentalternative of hierarchical clustering algorithms may use specialratepurposes.

(c) SOM (Self-Organization Map)

Stimulated by neural networks in the brain, SOM uses anopposition and collaboration mechanism to attain unsupervised learning. In the traditional SOM, a set of nodes isapproved in anarithmetic pattern, classically 2-dimensional pattern. Every node is relatedto a weight vector with the identicalaspect as the input space. The intention of SOM is to locate a superior mapping from the high dimensional input space to the 2-D depiction of the nodes. One approach to using SOM for clustering is to observe the items in the input space 
signify by the same node as assembled into a cluster. All through the training, every object in the input is accessible to the map and the best identical node is identified.

\section{(d) Expectation-maximization Clustering}

The EM algorithm is an allocation-based clustering algorithm. Distribution-based clustering algorithms suppose that objects are formed according to a prospectsupply. Special clusters can be measured created according to different possibility distributions. For each entity, themostprobability of an object belonging to anexplicit cluster is figured. A number of clusters, $\mathrm{k}$, isresoluteproceeding to clustering. EM is measured one of the most admired distribution-based clustering algorithms.

\section{Feature Selection}

There are someconditions that can delay the development of feature selection, such as the existence of unrelated and unnecessary features, noise in the data or interfaceamong attributes. In the presence of hundreds or thousands of features, such as DNA microarray investigation, researchers perceivethat is general that a huge number of features is not revealingsince they are either unrelated or unnecessary with esteem to the class perception. Furthermore, when the number of features is high excluding the number of examples is undersized, machine learning gets mainlycomplexbecause the search space will be lightlyinhabited and the reproduction will not be capableof distinguishingproperly thesignificant data and the noise $[9,10]$.

In addition,the classification, feature selection techniques can also be categorized into three methods: filters, wrappers, and embedded techniques. By means of such anenormous body of feature selection methods, the demandoccurs to find out various criteria that permit users to sufficientlymake a decision which algorithm to use in assured situations [7]. This work evaluates several feature selection techniques in the prose and ensures their concert in a simulatedprohibited experimental situation, distinct the capacity of the algorithms to choose the appropriate features and to get rid of the irrelevant ones not including authorizing noise or redundancy to hinder this development.

Table 3: Feature Selection Methods Summary

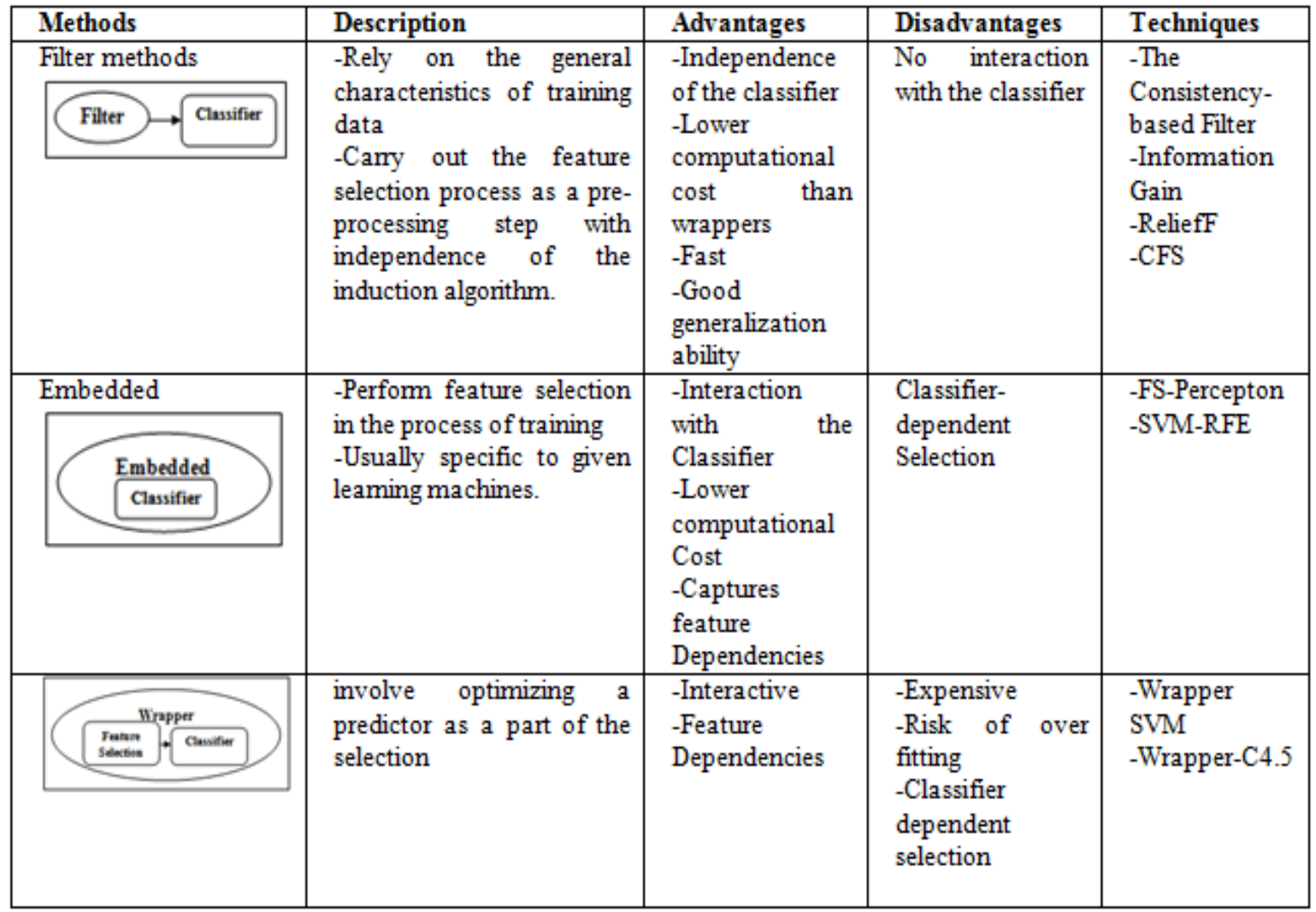

Table 3 provides a summary of the characteristics of the three feature selection techniques, representing the mainly prominent advantages and disadvantages, as fine as various examples of everymethod that will be promoteexplicated. Within filters, one can discriminateamongunivariate and multivariateprocesses [13]. Univariatetechniques are prompt and scalable, althoughdisregard feature dependencies. On the other hand, multivariate filters customs feature dependencies, but at the rate of being slower and take away scalable than univariate methods. 
Table 4: Feature Selection Methods Evaluation

\begin{tabular}{|c|c|c|c|}
\hline Techniques & Description & Advantages & Disadvantages \\
\hline Information Gain(IG) & $\begin{array}{l}\text {-This filter provides an } \\
\text { ordered ranking of all } \\
\text { the features and a } \\
\text { threshold value is } \\
\text { required } \\
\text {-Univariate filter } \\
\text { method }\end{array}$ & $\begin{array}{l}\text {-Scalable } \\
\text {-Fast } \\
\text {-Classifier independent }\end{array}$ & $\begin{array}{l}\text { Ignores feature } \\
\text { dependencies }\end{array}$ \\
\hline $\begin{array}{l}\text { Correlation-based feature } \\
\text { selection (CFS) }\end{array}$ & $\begin{array}{l}\text {-That ranks feature } \\
\text { subsets according to a } \\
\text { correlation-based } \\
\text { heuristic evaluation } \\
\text { function. } \\
\text {-Multivariate filter } \\
\text { method }\end{array}$ & $\begin{array}{l}\text {-Redundant features should be } \\
\text { screened out } \\
\text { - Irrelevant features should be } \\
\text { ignored } \\
\text {-Faster computation }\end{array}$ & $\begin{array}{l}\text { Classifier- } \\
\text { independent } \\
\text { Selection }\end{array}$ \\
\hline $\begin{array}{ll}\text { mRMR } & \text { (minimum } \\
\text { Redundancy } & \text { Maximum } \\
\text { Relevance) } & \end{array}$ & $\begin{array}{l}\text { Selects features } \\
\text { that have the highest } \\
\text { relevance with the } \\
\text { target class and are also } \\
\text { minimally redundant }\end{array}$ & $\begin{array}{l}\text { - Eliminates redundancy } \\
\text {-Tests the relevance of genes } \\
\text { in combination with other } \\
\text { genes }\end{array}$ & $\begin{array}{l}\text { Time complexity } \\
\text { more as compared } \\
\text { to filter methods }\end{array}$ \\
\hline $\begin{array}{l}\text { Embedded methods } \\
\text { (SVM-RFE Recursive } \\
\text { Feature Elimination for } \\
\text { Support Vector Machines, } \\
\text { FS-P Feature Selection- } \\
\text { Perceptron) }\end{array}$ & $\begin{array}{l}\text {-SVM embedded } \\
\text { method performs } \\
\text { feature selection by } \\
\text { iteratively training a } \\
\text { SVM classifier with the } \\
\text { current set of features } \\
\text { - A perceptronis a type } \\
\text { of artificial neural } \\
\text { network that can be } \\
\text { seen as the simplest } \\
\text { kind of feed-forward } \\
\text { neural network }\end{array}$ & $\begin{array}{l}\text {-Tests the predictivepower of } \\
\text { genes } \\
\text {-Less computational } \\
\text { complexity compared to } \\
\text { wrapper method } \\
\text {-Less prone to over-fitting }\end{array}$ & $\begin{array}{l}\text { Heavily dependent } \\
\text { on the model, so } \\
\text { they can fail to fit } \\
\text { the data well }\end{array}$ \\
\hline $\begin{array}{l}\text { Wrapper methods } \\
\text { (SVM, Wrapper-C4.5) }\end{array}$ & $\begin{array}{l}\text { Evaluates attribute sets } \\
\text { by using a learning } \\
\text { scheme. Cross- } \\
\text { validation is used to } \\
\text { estimate the accuracy of } \\
\text { the learning scheme for } \\
\text { a set of attributes. }\end{array}$ & $\begin{array}{l}\text { Carries out exhaustive search, } \\
\text { generating optimal solutions }\end{array}$ & $\begin{array}{l}\text {-Exponential time } \\
\text { complexity } \\
\text {-Complex } \\
\text {-Doesn't take } \\
\text { enough measures } \\
\text { to eliminate } \\
\text { redundancy } \\
\text {-Prone to } \\
\text { Over-fitting }\end{array}$ \\
\hline
\end{tabular}

While filter techniques indulgence the difficulty of finding a good feature separationseparately of the formcollection step, wrapper methods implant the formsuggestionexploration within the feature subset search. In this association, a search process in the space of probably feature subsets is distinct, and assorted subsets of features produce and estimated. The assessment of a precise subset of features is acquired by training and testing a detailed classification model, interpretation this approach customized to a specific classification algorithm [13, 18]. Though, as the break of feature subsets develops exponentially with a number of features, heuristic search techniques are used to conduct the search for thebest subset. These explore methods can be separatedinto two classes: deterministic and randomized search algorithms.

\section{Classification}

Classification segregatesdata sectionsinto objective classes. The classification methodforecaststhe intention set for every data points. For instance, thepatient can be classified as ahighthreator low threat patient on the source of their disease prototype using data classification approach. It is a supervised learning approach having known class categories. Binary and multi-level are the two techniquesof classification. [16]. Dataset is divided as training and testing dataset. By means of training dataset trained the classifier. The exactness of the classifierpossibly will be tested using test dataset. Hu et al. used special classification scheme such as decision tree, SVM and ensemble approach for investigating microarray data [17]. Further,utilize of theclassifier in thehealth field is discussed by Haticeet al., to analysis the skin diseases with weighted KNN classifier [22].

The survey work exposed that there is no only best algorithm which defersimprovedeffect for each dataset. Classification methods are also used for expecting the behaviorrate of healthcare services which is enhanced with speedydevelopment every year and is fetching a keyanxiety for everyone. Following are the various categorization algorithms used in healthcare: 
Table 5: Classification Techniques Evaluation Table

\begin{tabular}{|c|c|c|}
\hline Techniques & Advantages & Disadvantages \\
\hline K-NN & $\begin{array}{l}\text { It is easy to implement. } \\
\text { Training is done in faster manner. }\end{array}$ & $\begin{array}{l}\text { 1. It requires large storage } \\
\text { Space. } \\
\text { 2. Sensitive to noise. } \\
\text { 3. Testing is slow. }\end{array}$ \\
\hline Decision Tree & $\begin{array}{l}\text { It minimizes the ambiguity of } \\
\text { complicated decisions and } \\
\text { assigns exact values to outcomes } \\
\text { of various actions. } \\
\text { It can easily process the data with } \\
\text { high dimension. } \\
\text { It is easy to interpret. }\end{array}$ & $\begin{array}{l}\text { 1. It is restricted to one output } \\
\text { attribute. } \\
\text { 2. It generates categorical } \\
\text { Output. } \\
\text { 3. It is an unstable classifier i.e. } \\
\text { performance of classifier is } \\
\text { depend upon the type of dataset. } \\
\text { 4. If the type of dataset is } \\
\text { numeric than it generates a } \\
\text { complex decision tree }\end{array}$ \\
\hline Support Vector Machine & $\begin{array}{l}\text { 1. Better Accuracy as compare to } \\
\text { other classifier. } \\
\text { 2. Easily handle complex nonlinear } \\
\text { data points. } \\
\text { 3. Over fitting problem is not as } \\
\text { much as other methods. }\end{array}$ & $\begin{array}{l}\text { 1. Computationally expensive. } \\
\text { 2. The main problem is the } \\
\text { selection of right kemel function. } \\
\text { For every dataset different kemel } \\
\text { function shows different results. } \\
\text { 3. As compare to other methods } \\
\text { training process take more time. }\end{array}$ \\
\hline Neural Network & $\begin{array}{l}\text { 1. Easily identify complex } \\
\text { Relationships between dependent } \\
\text { and independent variables. } \\
\text { 2. Able to handle noisy data. }\end{array}$ & $\begin{array}{l}\text { 1. Local minima. } \\
\text { 2. Over-fitting. } \\
\text { 3. The processing of ANN network } \\
\text { is difficult to interpret and require } \\
\text { High processing time if there are } \\
\text { large neural networks. }\end{array}$ \\
\hline Bayesian Belief Network & $\begin{array}{l}\text { 1. It makes computations process } \\
\text { easier. } \\
\text { 2. Have better speed and accuracy } \\
\text { for huge datasets. }\end{array}$ & $\begin{array}{l}\text { 1. It does not give accurate } \\
\text { Results in some cases where there } \\
\text { exists dependency among variables. }\end{array}$ \\
\hline
\end{tabular}

(a) K-Nearest Neighbour (K-NN)

K-Nearest Neighbour (K-NN) is one of the simplest classifiersthat notices the unrevealed data endby means of the previously recognized data pointsand confidential data points according to the selection system. K-NN orders the data points using additional than one nearest neighbor. K-NN has a number offunctions in dissimilarregions such as health datasets, image domain, cluster study, pattern identification, online advertising etc.Shuman et al. used K-NN classifier for investigating the patients suffering from heart disease. The statistics werecomposedof UCI and experimentation was executed using exclusive ofselection or with selection K-NN classifier and it is established that K-NN attainsimprovedexactness without selection in theanalysis of heart diseases as evaluate towith selection K -NN.

\section{(b) Decision Tree (DT)}

DT is related tothe flowchartin which all non-leaf nodes standfor ananalysis on aparticular feature and every divisionrepresents aconclusion of that test and each leaf node hasa category label. The node at the topmost labels in the tree is known to be theroot node. Constructing a decision for everydifficulty doesn't require anykind of field knowledge. Decision Trees is a classifier that uses treesimilar to thegraph. Khan et al., used decision tree for forecasting the survivability of breast cancer patient and Chien et al., proposed a widespread hybrid decision tree classifier for categorizing the movement of apatient having persistent disease.

(c) Support Vector Machine (SVM)

The support vector machine(SVM) classifier generatesanoverexcitedlevel surface or severalhyperplanes in thehigh dimensional gap that is valuable for classification, degeneration and otherproficientresponsibilities. SVM havelots ofattractive features appropriate to this it is gaining esteem and has acapableexperimentalrecital. SVM buildsanoverexcitedlevel surface in unique input space to split the data ends. An amount of time it is complicated to achievepartition of data points in original input space, so to constructpartition easier the innovativerestricted dimensional space plotted intooriginalprivileged dimensional space. 
Soliman et al. used SVMcategorization approach for classification of a range of diseases and SVM mutually with k-means clustering werefunctional on microarray data for recognizingthe diseases. SVM is one of the most accepted approaches that are used by the researcher in healthcare domain for classification.

\section{(d) Neural Network (NN)}

This is an algorithmfor categorization that utilizesascentdecline methodand based on abiologicalanxious systemhaving numerousconsistenthandingoutessentialsidentified as neurons, carryingout inconcord tocrackexplicitdifficulty. Regulationsare extortedfrom theeducated Neural Network (NN) assistin developing interoperability of the educated network. To resolveaparticulardifficulty $\mathrm{NN}$ used neurons which are structuredhanding out elements. Neural Network is used for classification and model recognition. An NN is adaptive innature sinceit alters itsformation andregulates itspower inorder toreducethe fault.A collection neural network method is projected by Das et al., for analysis of heart disease in organize to extend theefficientdecision support system.

\section{(e) Bayesian Methods}

The classification based on Bayes premise is acknowledgedasBayesian classification. It is astraightforward classifier which is accomplished by using classification algorithm. Bayes theorem suppliessource for Naive Bayesian Classification and Bayesian Belief Networks (BBN). The majorcrisiswith Naïve Bayes Classifier is thatit supposedthat allcharacteristics areself-regulatingwith eachother whereasin medicalfield attributessuch as patientsymptoms and their fitnessstatus are interrelated with each other.Bayesian Belief Network is extensively used bynumerous researchers in thehealthcarearea.

\section{Association Mining}

Gene expression data necessitates a few steps of data processing prior to it can be investigated for association rules. In market basket analysis an entry is either obtained or not obtained but microarray data includes of uninterrupted statistical data. The initial step is to discretize the data, renovate it to a Boolean or tertiary record. The majorityof the request of mining association ruleon microarraygene expressionstill relieson discretization responsibilitiespriortopertaining anydata mining method.The standardized microarray dataset is frequentlysignifying as sequences of constant numbers [19]. Discretization is the progression of conversion from continuous data into distinct data.

The threshold process used to discretize the information. This technique is appropriate for microarray study. Genes with chronicle expression values superior toafastidious value are considered as overexpressed, if not as below expressed. By means of threshold method, every gene expression is transformed into one of the two separate values 1, 0 for over-expressed and under-expressed. Association rules are asignificant group of techniques of pronouncement samples in data. Association rule mining method remove appealing associations along with acollection of items (genes) in ahuge quantity of information. One of the most illustrious purposes of these techniques is market basket analysis $[19,20]$ where the key goal is to discover interactions between the acquired items under different operation. An association rule is functional on microarray dataset in regulate to locate the associations between genes under different illustration.

\section{Conclusion}

In this paper, we analyzed various image analysis techniques used in bioinformatics sector to identify and classify the diseases using microarray data. The system based on association mining and classification for categorize the diseases from the defected genes.In connection with this, important phases such as microarray data pre-processing, clustering, feature selection, classification and association mining. Here microarray data is attained by bioinformatics then pre-processed using normalization and summarization. Pre-processed data are further used to analyze the expression data using expression analysis tests such as statistical tests, U-test. The analyzed gene expression is clustered using clustering techniques like K-means, SOM or Hybrid method. The feature selection is themain objective to predict the various diseases using this gene expression. Finally, thethreshold value is applied on association rule mining to predict and classify the diseases using microarray data. One can extend this work to generate most significant feature vectors for efficient and accurate classification.

\section{References}

[1] Jun S. Liu Department of Statistics Harvard University "Bioinformatics: Microarrays Analyses and Beyond".

[2] AlirezaOsareh andBitaShadgar "Microarray Data Analysis forCancer classification" IEEE Antalya, Turkey 2009, pp.125-132.

[3] R. D. Canlas Jr., "Data Mining in Healthcare:Current Applications and Issues", (2009).

[4] H. C. Koh and G. Tan, "Data Mining Application in Healthcare", Journal of Healthcare Information Management, vol. 19, no. $2,(2005)$

[5] F. Hosseinkhah, H. Ashktorab, R. Veen, M. M. Orange O., "Challenges in Data Mining on Medical Databases", IGI Global, (2009), pp. 502-511.

[6] K. Srinivas, B. KavithaRani, and Dr. A.Govrdhan, "Applications of Data Mining Techniques in Healthcare and Prediction of Heart Attacks", International Journal of Computer Science and Engineering, vol. 02 no. 02, (2010), pp. 250-255.

[7] C. Shang and Q. Shen, "Aiding classification of geneexpression data with feature selection: a comparative study." vol. 1, 2005, pp. 68-76. 
[8] Piyushkumar A. Mundra andJagath C. Rajapakse "Support Vectors Based Correlation Coefficient for Gene and SampleSelection in Cancer Classification" IEEE 2010.

[9] YvanSaeys, InakiIn and Pedro Larranaga "A reviewof feature selection techniques in bioinformatics" 2005 pp 1-10.

[10] K Blekas, Nikolas P. Galatsanos, andIoannis Georgiou, "an unsupervised artifact correction approach for the analysis of DNA microarray images", IEEE, 2003.

[11] Jianhua Xuan, Eric Hoffman, Robert Clarke, Yue Wang, "Normalization of Microarray Data by Iterative Nonlinear Regression", IEEE conference on IBBE, 2005.

[12] Bhanot,G .,et al. (2006) A robust meta-classification strategy for cancer detection from M S data.Proteomics, 6, 592-604

[13] Blanco,R., et al. (2004) Gene selection for cancer classification using wrapper approaches. Int.J. Pattern Recognition. Artif. Intell. , 18, $1373-1390$.

[14] Dudoit,S., et al. (2002), Comparison of discriminant methods for the classification of tumors using gene expression data. J. Am. Sta t. A stock., 97, 77-87.

[15] Alon, U., Barkai, N., Notterman, D., Gish, K., Ybarra, S., Mack, D., Levine, A., - Broad patterns of gene expression revealed by clustering analysis of tumor and normal colon tissues probed by oligonucleotide arraysll, Proc. Natl. Acad. Sci., 1999, 96 (12), $6745-$ 6750.

[16] Antonov, A.V., Tetko, I.V., Mader, M.T., Budczies, J., Mewes, H.W., -Optimization models for cancer classification: extracting gene interaction information from microarray expression datall, Bioinformatics 20, 2004, 644-652.

[17] A. Statnikov, C.F. Aliferis, I. Tsamardinos, D. Hardin, S. Levy, -A comprehensive evaluation of multicategory classification methods for microarray gene expression cancer diagnosis, B Bioinformatics, Vol. 21, 2005, No. 5, pp 631-643.

[18] A. E. Akagi, A. Amine, A. E. Ouardighi, D.Aboutajdine, -Feature selection for Genomic data by combining filter and wrapper approachesll, INFOCMP Journal of computer science,2009, vol. 8, no. 4, pp. 28-36.

[19] Eisen MB, Spellman PT, Brown PO, Botstein D. Cluster analysis and display of genome-wide expression patterns. Proc Natl AcadSciUSA 1998;95:14863-8.

[20] AzadehMohammadi, Mohammad H Saraee, MansoorSalehi, "Identification of disease-causing genes usingmicroarray data mining and Gene Ontology" Mohammadi et al. BMC Medical Genomics 2011.

[21] Shang Gao, Omar Addam and colleagues, "Robust Integrated Framework for Effective Feature Selection and Sample Classification and Its Application to Gene Expression Data Analysis" IEEE 2012 pp. 112-119.

[22] One Huey Fang, Norwati Mustapha, Md. Nasir Sulaiman "Integrating Biological Information for Feature Selection in Microarray Data Classification" 2010 Second International Conference on Computer Engineering and Applications IEEE, pp. 330-334. 